\title{
Comparison of Tir from enterohemorrahgic and enteropathogenic Escherichia coli strains: two homologues with distinct intracellular properties
}

\author{
Chen-Hua Chuang, Hao-Jie Chiu, Sheng-Chieh Hsu, Jin-Yuan Ho \& Wan-Jr Syu* \\ Institute of Microbiology and Immunology, National Yang-Ming University, 155 Sec. 2, Li-Noong St., Beitou, \\ Taipei, 112, Taiwan
}

Received 2 April 2005; accepted 12 September 2005

(C) 2005 National Science Council, Taipei

Key words: EHEC, EPEC, F-actin, pathogenesis, pedestal, Tir

\begin{abstract}
Summary
Tir of enteropathogenic Escherichia coli (EPEC) or enterohemorrahgic E. coil (EHEC) is translocated by a type III secretion system to the host cell membranes where it serves as a receptor for the binding of a second bacterial membrane protein. In response to the binding, EPEC Tir is phosphorylated at $\mathrm{Tyr}_{474}$, and this phosphorylation is necessary for the signaling of pedestal formation. Tir of EHEC has no equivalent phosphorylation site but it is similarly needed for cytoskeleton rearrangement. How these two Tir molecules achieve their function by apparently different mechanisms is not completely clear. To examine their intrinsic differences, the two Tirs were expressed in HeLa cells and compared. Actin in complexes could be pelleted down from the lysate of cells expressing EHEC Tir but not EPEC Tir. By immunostaining, neither Tir molecule was found in phosphorylated state. In the cytoplasm, EHEC Tir was frequently found in fibrous structures whereas EPEC Tir was observed completely in a diffusive form. The determinant critical for the EHEC Tir fibrous formation was mapped to the C-terminal region of the molecule that deviates from the EPEC counterpart. This region may play a role in taking an alternative route different from $\mathrm{Tyr}_{474}$ phosphorylation to transduce signals.
\end{abstract}

\section{Introduction}

Enterohemorrhagic Escherichia coli (EHEC) and enteropathogenic E. coli (EPEC) are among the major etiological agents for human diarrhea [1]. In spite of the virotype differences and variations in their virulence factors, these two microbes do share one common pathogenic characteristic known as the attaching and effacing (A/E) intestinal lesion. $\mathrm{A} / \mathrm{E}$ lesions are characterized by the loss of microvilli and reorganization of the cytoskeletal structure [2] that contains condensed actin beneath the sites of bacterial attachment, termed the pedestal structure. While the mechanistic action

*To whom correspondence should be addressed. Tel.: + 886-228267112; Fax: +886-2-28212880; E-mail: wjsyu@ym.edu.tw of the $\mathrm{A} / \mathrm{E}$ lesion has not been completely understood, it is known that this pathogenic presentation is genetically linked to a pathogenicity island locating at the locus of enterocyte effacement (LEE) on both bacterial chromosomes [3, 4].

Away from their prophage sequences, the two LEEs are highly conserved and contain 41 open reading frames $[4,5]$. Among the better characterized products encoded by the LEE are Tir [6], intimin [7], and some of the type III secreted proteins including EspA [8], EspB [9], and EspD [10]. These are required for the complicated events of bacterial binding, the transferring of effectors, and the triggering of host signal transduction cascades during bacterial infection. While intimin remains on the bacterial surface, Tir translocates into the host cell and serves as a receptor for the 
intimin binding [6]. As the binding advances, Tir along with EspF [11] and MAP [12] serves as effector proteins to affect multiple cellular processes including cytoskeletal rearrangement and apoptosis.

In characterized EPEC infection, Tir is phosphorylated at residue $\mathrm{Tyr}_{474}$ after its translocation into host cells [13]. This tyrosine phosphorylation is required for the interaction of EPEC Tir with cytokeratin-18 [14] and for the localization of Grb2 and CrkII to the pedestals [15]. Nck, an adapter protein including Nck1 and Nck2, has the properties of binding to phosphorylated tyrosine in its Src homology 2 (SH2) domain and is involved in the activation of neural WiskottAldrich syndrome protein (N-WASP) in vitro to trigger actin polymerization [16]. Intriguingly, Nck has been found only in the EPEC-formed pedestals [17].

It has also been observed that N-WASP and Arp $2 / 3$ are localized to pedestals formed by EHEC $[15,18]$, and cells that lack N-WASP are resistant to pedestal formation by EHEC [19, 20]. Unlike Tir of EPEC, the EHEC counterpart does not contain an equivalent $\mathrm{Tyr}_{474}$, and Nck is not involved in the interaction event. To form a pedestal, apparently, the EHEC Tir must take an alternative strategy, which may rely on its molecular uniqueness. In this study, we examined the differences between the EHEC and EPEC Tir molecules when they were the only bacterial molecules directly produced in Hela cells. While no phosphorylation seemed to be identical for Tir from both origins, EHEC Tir in the cell lysates appeared to have some competency to stimulate actin polymerization that was not seen with EPEC. The appearances of the two Tir molecules in the cells also differed significantly. EHEC Tir was frequently found in fibrous structures whereas EPEC Tir was distributed diffusively in cytosol. These properties may explain, at least in part, why EHEC Tir could function differently from EPEC Tir $[19,21]$.

\section{Materials and methods}

\section{Bacterial strains and media}

EHEC strain ATCC 43888 was obtained from the American Type Culture Collection. An EPEC strain was obtained from Center of Disease Control, Taiwan, and its tir is identical to that of strain E2348/69 [4, 5] and has been confirmed by sequencing. Bacteria were grown at $37^{\circ} \mathrm{C}$ in LuriaBertani broth (Difco, Augsburg, Germany) or agar plates and supplemented with ampicillin $(100 \mu \mathrm{g} / \mathrm{ml})$ or chloramphenicol $(34 \mu \mathrm{g} / \mathrm{ml})$ when necessary.

\section{General recombinant DNA techniques}

Unless otherwise stated, restriction endonucleases, DNA-modifying enzymes and polymerases were purchased from New England Biolabs (Beverly, MA, USA). DNA manipulation procedures were performed either as described [22] or as recommended by the manufacturers. DNA was purified from a mini-column (Qiagen, Valencia, CA, USA), analyzed by restriction enzyme digestion and finally confirmed by sequencing. Sequencing was carried out routinely by a contract service from Mission Biotech (Taipei, Taiwan, ROC).

\section{Plasmids construction}

The entire tir gene was PCR amplified using the primer pairs NTir-F/NTir-R(Pst $)$ and NTir-F/ NTir-PR (EcoRI) (Table 1) from the chromosomal DNA of EHEC or EPEC. The EHEC-tir amplicon was digested with PstI and cloned into $S m a \mathrm{I} / P s t \mathrm{I}-$ digested pCMV-DD6 and pCMV-DS [23] to create $\mathrm{pC} / \mathrm{d}-\mathrm{Tir}-\mathrm{m}-\mathrm{H}$ and $\mathrm{pC} / \mathrm{d}-\mathrm{Tir}-\mathrm{H}$, respectively. The EPEC-tir amplicon was digested with EcoRI and cloned into SmaI/EcoRI-restricted pCMV-DD6 to create $\mathrm{pC} / \mathrm{d}$-Tir-m-P. To easily follow these clones, a " $\mathrm{d}$ " is incorporated into the name of a plasmid to indicate that there is a D epitope engineered at the $\mathrm{N}$-terminus of the Tir construct whereas a built-in " $\mathrm{m}$ " stands for an M epitope engineered at the Tir C-terminus [23]; " $\mathrm{H}$ " and " $\mathrm{P}$ " represent the origin of Tir deriving from EHEC and EPEC, respectively. Thus, as an example, HeLa cells transfected with $\mathrm{pC} / \mathrm{d}-\mathrm{Tir}-\mathrm{m}-\mathrm{P}$ would produce a construct named d-Tir-m-P. This product is identical to EPEC Tir except for that both ends of the molecule are tagged with $\mathrm{D}$ and $\mathrm{M}$ epitopes, respectively.

Plasmid pC/d-Tir-m-H was digested with $X b a \mathbf{I} / N r u \mathrm{I}$, treated with the Klenow fragment of DNA polymerase I and self-ligated to result in $\mathrm{pC} /$ Tir-m-H. To create $\mathrm{pC} / \mathrm{d}-\mathrm{Tir}_{\mathrm{H} 1-390}$, pC/d-Tir$\mathrm{m}-\mathrm{H}$ was annealed with primers NCMV5 and 
Table 1. Primers used for PCR.

\begin{tabular}{ll}
\hline Primer & Sequence $\left(5^{\prime} \rightarrow 3^{\prime}\right)$ \\
\hline NCMV5 & CACCGGGACCGATCCAGCCTGAC \\
NT7 & TAATACGACTCACTATAGGG \\
NTir-F & TTATGCCTATTGGTAACCTTGG \\
NTir-F $($ AflIII $)$ & CCCACATGTCTATTGGTAACCTTG* \\
NTir-R $($ Pst $\mathrm{I})$ & CAGCTGCAGACGAAACGATGGGATCCCGG \\
NTir-R $($ HindIII $)$ & CCCAAGCTTAGACGAAACG \\
NTir-PR $($ Eco $\mathrm{RI})$ & TAGAATTCACGAAACGTACTGGTCCCGG \\
NTir-1158R & CCGGCTGATTT \\
NTir-680F $($ Bam HI $)$ & GCGGATCCCAGATCCTAAA \\
NTir-1560R $($ HindIII $)$ & CCCAAGCTTACGCATAAGTGCTTTGAATCCC \\
NTir $(H 536 P) F$ & ATGGGAGGATTAACG \\
NTir(H536P $)$ R & CGTTAATCCTCCCAT \\
Lee-22239 & CAGAAAGTTGATGAGCAGTGCGGC \\
NTir-171R & TATCAATCAGCCATAGAATTCC \\
\hline
\end{tabular}

NTir-1158R and PCR amplified. The PCR product was digested with BamHI and cloned into SmaI/BamHI-digested pCMV-DD6. To generate $\mathrm{pC} / \mathrm{d}-\mathrm{Tir}_{\mathrm{H} 228-390}, \mathrm{pC} / \mathrm{d}-\mathrm{Tir}-\mathrm{m}-\mathrm{H}$ was primed with NTir-680F(BamHI) and NTir-1158R, and the PCR product was digested with BamHI and subsequently cloned into SmaI/BamHI-restricted pCMV-DD6. To create $\mathrm{pC} / \mathrm{d}-\mathrm{Tir}_{\mathrm{H} 228-558}-\mathrm{m}$, the DNA segment was PCR amplified from $\mathrm{pC} / \mathrm{d}$-Tir$\mathrm{m}-\mathrm{H}$ with primers NTir- $680 \mathrm{~F}($ Bam $\mathrm{HI})$ and NTir$\mathrm{R}(P s t \mathrm{I})$. The amplicon was digested with BamHI and cloned into SmaI/BamHI-treated pCMVDD6. The resulting plasmid was subsequently cleaved with $S m a \mathrm{I} / K p n \mathrm{I}$ and ligated with $S m a \mathrm{I} /$ $\mathrm{KpnI}$-digested tir fragment from $\mathrm{pC} / \mathrm{d}-\mathrm{Tir}-\mathrm{m}-\mathrm{H}$. To create $\mathrm{pC} / \mathrm{d}-\mathrm{Tir}_{\mathrm{H} 497-558}$, pCMV-DD6 was cleaved with $S m a \mathrm{I} / K p n \mathrm{I}$ and ligated with $S m a \mathrm{I} /$ $K p n I$-digested tir fragment from $\mathrm{pC} / \mathrm{d}-\mathrm{Tir}-\mathrm{m}-\mathrm{H}$.

Chimeric Tir molecules comprising fragments derived from EHEC Tir and EPEC Tir were constructed by a two-round PCR procedure. According to the alignment in Figure 1, the fusion junctions were chosen in regions where the coding nucleotides are conserved in both EHEC and EPEC. For clarity of nomenclature, the chimeras are designated as follows. $\operatorname{Tir}_{\mathrm{H} 536 \mathrm{P}}$ indicates that the chimeric Tir carries residues $1-536$ originated from EHEC Tir and those residues after 536 are derived from EPEC Tir. Similarly, $\operatorname{Tir}_{\mathrm{P} 528 \mathrm{H}}$ represents residues 1-528 from EPEC Tir and residues 529-558 from EHEC Tir. To construct these chimeras, the strategies used were basically identical except for the paired primers. Taking generation of $\mathrm{pC} / \mathrm{d}-\mathrm{Tir}_{\mathrm{H} 536 \mathrm{P}}-\mathrm{m}$ as an example, NCMV5 was paired with NTir(H536P)R to amplify the $5^{\prime}$ portion of EHEC tir from $\mathrm{pC} / \mathrm{d}$ Tir-m-H, and NTir(H536P)F was paired with NT7 to amplify the $3^{\prime}$ portion of EPEC tir from pC/d-Tir-m-P. Because NTir(H536P)F and NTir (H536P)R are complementary, the above two PCR products overlap partially in sequence. Denaturation and annealing the PCR products followed by polymerase extension yielded a complete chimeric template, from which amplified chimeric product was obtained by a second PCR that used primers NCMV5 and NT7. The product, digested with $X b a \mathrm{I}$ and $P s t \mathrm{I}$, was then subcloned into the $X b a \mathrm{I} /$ Pst I-digested pCMV-DD6. The resulting plasmid

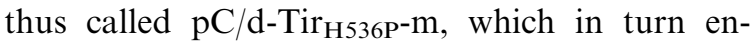
codes the chimeric protein $\mathrm{d}-\mathrm{Tir}_{\mathrm{H} 536 \mathrm{P}-\mathrm{m}}$.

To create $\mathrm{pQE60-Tir-H,} \mathrm{a} \mathrm{DNA} \mathrm{segment} \mathrm{was}$ PCR amplified with primers NTir-F(AflIII) and NTir-R(HindIII) from pC/d-Tir-m-H and digested with $A f$ III/HindIII. The resulting DNA was cloned into $N c o \mathrm{I} / H i n d I I I-d i g e s t e d ~ p Q E 60$. To create $\mathrm{pQE} 60-\mathrm{Tir}_{\mathrm{H} 1-521}$, a DNA segment was PCR amplified with primers NCMV5 and NTir1560R (HindIII) from pC/d-Tir-m-H and digested with $S p h \mathbf{I} / H$ indIII. The resulting DNA was cloned into SphI/HindIII-digested pQE-Tir-H.

\section{Cell culture and transfection}

HeLa cells were maintained in DMEM containing $10 \%$ fetal calf serum and appropriate antibiotics at 


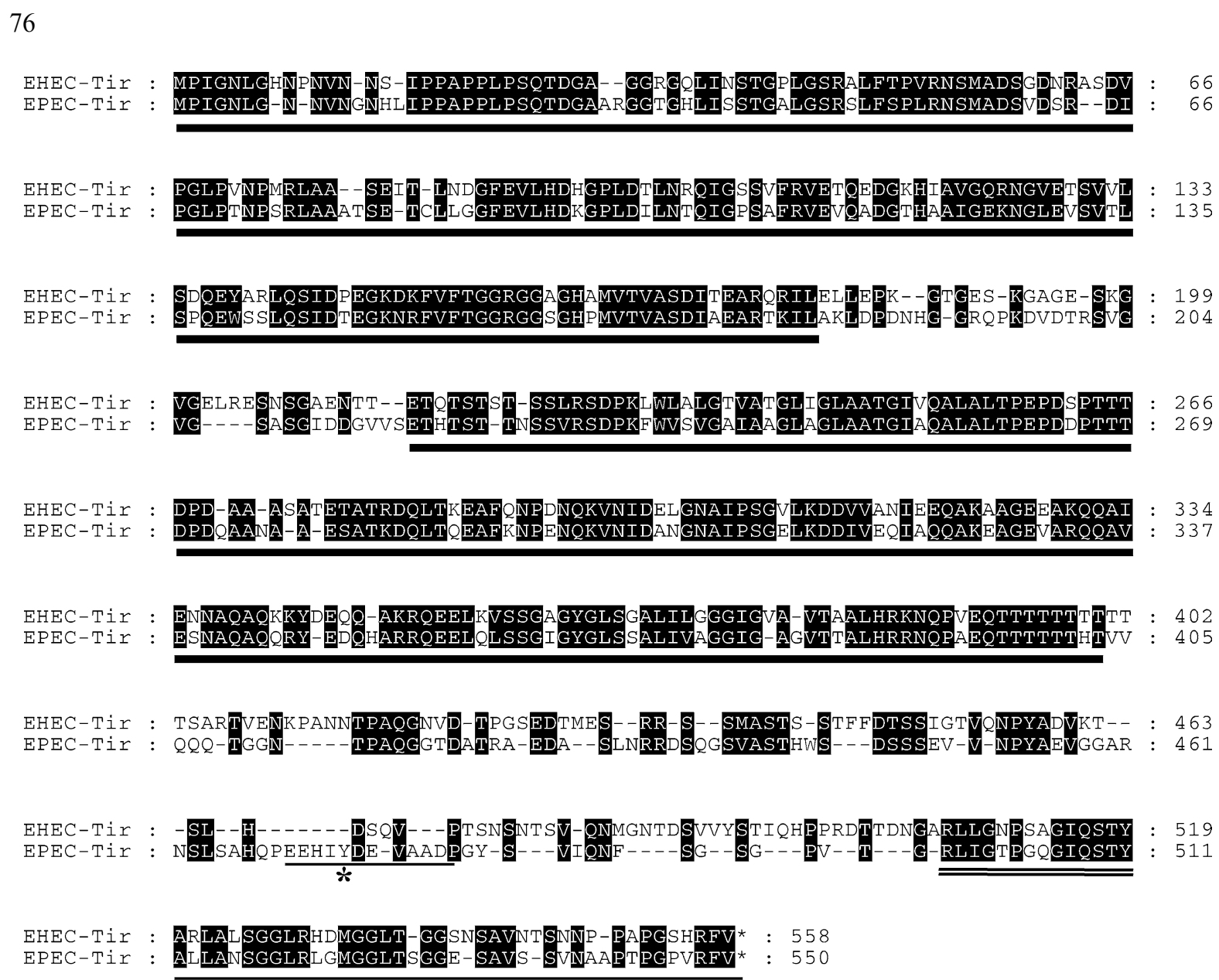

Figure 1. Amino acid sequence alignment of EHEC Tir and EPEC Tir. Identical residues are shaded while gaps were introduced to maximize the alignment and these are represented by dashes. $\mathrm{Tyr}_{474}$ is marked by an asterisk while the 12-residue phosphopeptide [24] capable of triggering focused actin assembly is underlined. Conservative regions are indicated underneath the sequences: amino acid identity over $76 \%$, thick line; $66 \%$ identity, double lines.

$37^{\circ} \mathrm{C}$ in a $5 \% \quad \mathrm{CO}_{2}$ incubator. For transient expressions, cells were grown on 100-mm dishes overnight and then transfected with $20 \mu \mathrm{g}$ DNA using the calcium phosphate method previously described [25]. After transfection, cells were cultured for additional $48 \mathrm{~h}$ before analysis.

\section{Western blotting}

Anti-RGS-His tag monoclonal antibody (MAb) was obtained from Clontech (Palo Alto, CA, USA). Anti-PY MAb was purchased from Sigma (St. Louis, MO, USA). Anti-D MAb (HP6A1) that binds to the $\mathrm{D}$ epitope and anti-M MAb (SC1D7) against the $M$ epitope have been described previously [23]. Anti-EHEC-Tir was prepared by immunizing mice with a recombinant EHEC Tir that had been purified by affinity binding to a nickel ion column followed by elution from a polyacrylamide gel. This antibody only recognized EHEC Tir and did not cross-react with Tir from EPEC in both Western blotting and immunofluorescence staining.

Western blotting analysis was carried out as previously described [26]. In the immunoreactions, antibodies were diluted with $5 \%$ skimmed milk in Tris-buffered saline (TBS) and added to the nitrocellulose membranes. After appropriate incubation and washing, the membranes were reacted with goat anti-mouse $\operatorname{IgG}$ antibodies conjugated with horseradish peroxidase (HRP) (Sigma). Finally, the membranes were developed using Renaissance Western Blot Chemiluminescence Reagent Plus (NEN Life Science, Boston, MA, USA), and the signals were detected by X-ray film exposure. 


\section{Immunofluorescence staining}

Immunofluorescence staining was carried out by a slight modification of the methods described [27]. For infections, subconfluent HeLa cells on glass cover slips were placed in the wells of 6-well culture plates containing $3 \mathrm{ml}$ of DMEM with $10 \%$ fetal calf serum. Overnight cultured bacteria were 1:100 diluted into the cell culture. After incubation at $37^{\circ} \mathrm{C}$ in a $5 \% \mathrm{CO}_{2}$ incubator for $5.5 \mathrm{~h}, 1-\mathrm{ml}$ fresh medium was added, and incubation was continued for additional $2.5 \mathrm{~h}$. When Tir was expressed from HeLa, cells were stained $48 \mathrm{~h}$ after transfection.

To stain proteins on the cell membrane, cells on cover slips were washed five times by gentle shaking in cold phosphate-buffered saline (PBS) and fixed with paraformaldehyde ( $4 \%$ in PBS) for $30 \mathrm{~min}$. To stain proteins intracellularly, cells were fixed with pre-cooled methanol/acetone $(1: 1 ; \mathrm{v} / \mathrm{v})$ for $5 \mathrm{~min}$. After fixation, the cells were washed again for three times with PBS.

To specifically stain the F-actin filaments, cells on the cover slips were treated with TRICTlabeled phalloidin (Jackson Lab, West Grove, Penn., USA) $(5 \mathrm{~g} / \mathrm{ml})$ in dilution buffer (PBS containing $1 \%$ bovine serum albumin). Bacteria were counterstained with rabbit anti-O157 antibody (Difco) followed by fluorescein isothiocyanate (FITC)-labeled anti-rabbit immunoglobulin (Jackson Lab). To stain DNA in cells, Hochest 33258 (Sigma) was used. The epitope-tagged Tir molecules were reacted with MAb HP6A1 or MAb SC1D7 [23]. Thereafter, an FITC-labeled antimouse immunoglobulin (Jackson Lab) was used to trace the mouse antibody bound on the targets. The stained cells on cover slips were examined using a fluorescence microscope. HeLa cells mock infected were similarly treated and used as a negative control. To calculate the frequencies of cells with Tir stained in condensed fibrous structures, cells were enumerated by counting at least 200 transfected cells in multiple randomly selected fields. Repeated experiments were carried out to ensure obtaining consistency.

\section{Insoluble complex formation and immunoprecipitation}

HeLa cells with or without the Tir expression were harvested in a lysis buffer $(10 \mathrm{mM}$ Tris-HC1
(pH 7.5) containing $10 \%$ glycerol, $0.5 \%$ Triton $\mathrm{X}-100,0.5 \%$ deoxycholic acid, $10 \mathrm{mM}$ PMSF, $40 \mathrm{mM} \mathrm{NaCl}, 1 \mathrm{mM} \mathrm{NaVO}, 1 \mathrm{mM} \mathrm{NaF}$ ). The cell lysates were clarified by centrifugation in a refrigerated centrifuge at $10,000 \mathrm{~g}$ for $10 \mathrm{~min}$. The supernatants collected were incubated at $4^{\circ} \mathrm{C}$ for $14 \mathrm{~h}$. After the incubation, the cloudy supernatants were centrifuged again, and the pellets collected were rinsed twice with the same buffer and dissolved in SDS sample buffer for protein analysis. Alternatively, Sepharose 4B beads (Sigma) coupled with protein A were suspended in $50 \mathrm{mM}$ Tris- $\mathrm{HCl}$ ( $\mathrm{pH} 7.5$ ) containing $150 \mathrm{mM}$ $\mathrm{NaCl}, 1 \mathrm{mM}$ EDTA, $0.25 \%$ gelatin, $0.1 \% \mathrm{NP}-40$, and $0.02 \% \mathrm{NaN}_{3}$ and mixed with MAb HP6A1 at $4^{\circ} \mathrm{C}$ for $4 \mathrm{~h}$ in a rotating drum. After washing three times with the same buffer, the protein A beads received $1 \mathrm{ml}$ of cell lysates in the lysis buffer. After further incubation for $14 \mathrm{~h}$ at $4{ }^{\circ} \mathrm{C}$, the whole sample was centrifuged for $20 \mathrm{~min}$ at $2500 \mathrm{~g}$ in a refrigerated centrifuge. The pellets were washed six times with the lysis buffer and finally suspended in the SDS sample buffer and boiled.

\section{ESI-Q-TOF mass spectrometry analysis}

The procedure for in-gel tryptic digestion was performed according to the method previously described [28] except for a slight modification. In brief, Coomassie blue stained protein spots excised from distilled water-washed gel were cut into small pieces in microfuge tubes. Gel pieces were dehydrated and destained with a mixture containing $50 \mathrm{mM}$ ammonium bicarbonate and $50 \%(\mathrm{v} / \mathrm{v})$ acetonitrile. The gels was then dried under vacuum and then swollen in sufficient volume of $10 \mathrm{mM}$ dithiothreitol in $25 \mathrm{mM}$ ammonium bicarbonate, then incubated at $56^{\circ} \mathrm{C}$ for $45 \mathrm{~min}$. After that, the gels were washed twice with $100 \mathrm{mM}$ ammonium bicarbonate, dehydrated with acetonitrile twice and dried under vacuum again. The digestion was carried out by swelling the gel at $4^{\circ} \mathrm{C}$ for $45 \mathrm{~min}$ in a sufficient volume of $50 \mathrm{mM}$ ammonium bicarbonate containing $5 \mathrm{mM} \mathrm{CaCl} 2$ and $12 \mathrm{ng} / \mu \mathrm{l}$ trypsin (Roche, Mannheim, Germany). The digestion was continued overnight at $37^{\circ} \mathrm{C}$. The gel was submitted to extraction with $20 \mathrm{mM}$ ammonium bicarbonate and three further extractions with $50 \%$ acetonitrile containing $5 \%$ trifluoroacetic acid. Pooled recovered peptides were concentrated under vacuum to 3-4 $\mu$ l and brought back up to 
$10 \mu \mathrm{l}$ in $0.1 \%$ trifluoroacetic acid. The sample was desalted on Zip Tip C18 microcolumns (Millipore, Bedford, MA, USA) before being loaded onto an ESI-Q-TOF mass spectrometer (Micromass, Beverly, MA, USA). Data from mass profiling were searched against the NR database by using Mascot (http://www.matrix-science.com).

\section{Generation of EHEC mutant with tir deleted}

To delete tir from the EHEC chromosome, the procedure previously described [29] was followed. In brief, the bacterial chromosomal DNA was used as the template for PCR amplification. Primers Lee-22239 and NTir-171R were used to amplify a fragment covering a $5^{\prime}$ flanking region and a small $5^{\prime}$ region of tir. The obtained PCR fragment was digested with BamHI. A second PCR using primers NTir-F and NTir-R(PstI) was performed to generate a fragment covering a $3^{\prime}$ segment of tir, and this PCR product was digested with BamHI and EcoRV. The above two PCR products were then three-way ligated into the BamHI-restricted Pinpoint Xa-3 (Promega, Madison, WI, USA), to result in Pinpoint-Xa3- $\Delta$ Tir. To construct pKO3- $\Delta$ Tir, Pinpoint-Xa3- $\Delta$ Tir was digested with $N o t \mathrm{I} / N r u \mathrm{I}$ and ligated into Not $\mathrm{I} /$ SmaI-digested pKO3. The resulting plasmid was electroporated into bacteria, and the bacteria with homologous recombination were then selected as described previously [30]. A so obtained mutant $(\Delta$ Tir) was examined for chromosomal deletion of tir by PCR using primers NTir-F and NTir$\mathrm{R}($ Pst $\mathrm{I})$ followed by confirmative sequencing of the PCR products.

\section{Results}

\section{Expression and immunostaining of Tir in cells}

To address why homologues differ adversely in molecular properties, the primary sequences of the two Tir proteins were first examined. As shown in Figure 1, EHEC Tir (558 residues) and that of EPEC (550 residues) have a long stretch of conserved sequence in the $\mathrm{N}$-terminal region and the middle region. The region covering residues $1-$ 180 (referring to the sequence of EHEC Tir) has a $76 \%$ amino acid identity whereas that covering residues $215-400$ has a $79 \%$ identity. The region spanning residues $506-558$ is conserved to a less extent, with an identity at $66 \%$. In contrast, residues 181-214 and residue 400-505 vary adversely between the two molecules. Also shown in Figure 1 is that the $\mathrm{Tyr}_{474}$ in EPEC Tir and its flanking sequences are not conserved at all in EHEC Tir.

EPEC Tir is translocated into the host cells as part of both the membrane and cytosol proteins after infection [6]. Similar cellular localizations have been found for EPEC Tir when transfected [24]. Whether EHEC is similarly distributed as EPEC Tir was closely examined. After transfection for $48 \mathrm{~h}$, the cells were fixed, and the Tir molecules were detected by immunofluorescence staining. The spotty patterns stained for Tir on the cell membrane appeared indistinguishable between those of EHEC and EPEC (data not shown). When the intracellular Tir was examined, a striking difference was observed (Figure 2a). EPEC Tir stained diffusely across the cytoplasm and intensely in the periphery surrounding the cell nucleuses. In contrast, EHEC Tir was stained in two different patterns; one was identical to that seen with EPEC Tir whereas a second (in $41 \%$ of the expression cells) was characterized by the appearance of thick fibrous structures. The staining patterns were similar (Figure 2a) irrespective of whether the anti-D (left panel) or anti-M (right panel) detections were used. Since the stained fibrous EHEC Tir structures resemble highly condensed actin filaments, simultaneous staining of the cells with anti-D for Tir and TRICTphalloidin for filamentous actin (F-actin) was performed. A typical result is shown in Figure $2 b$. Some of the signals for the EHEC Tir filaments were superimposable upon those of the F-actin, but most were not. Furthermore, it is worth noting that the actin filaments in the EHEC Tir-expressing cells were stained much thicker than those in the non-expressing cells (Figure 2b).

\section{Precipitated complexes stimulated by EHEC Tir}

Since the d-Tir-m-H fibrous structures were not necessarily co-localized with the condensed F-actin, it was then reasoned that the so produced EHEC Tir molecules might catalyze the fibrous structure formation of actin rather than there has been a direct association. To examine this possibility, HeLa cell lysates derived from the 
(a)

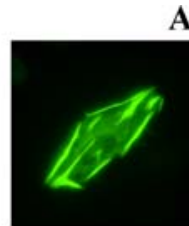

Anti-D
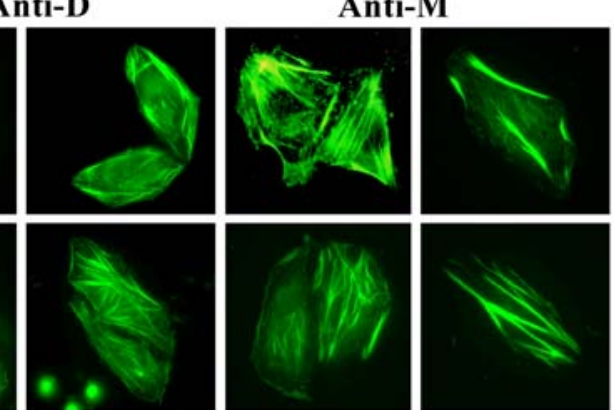

EHEC
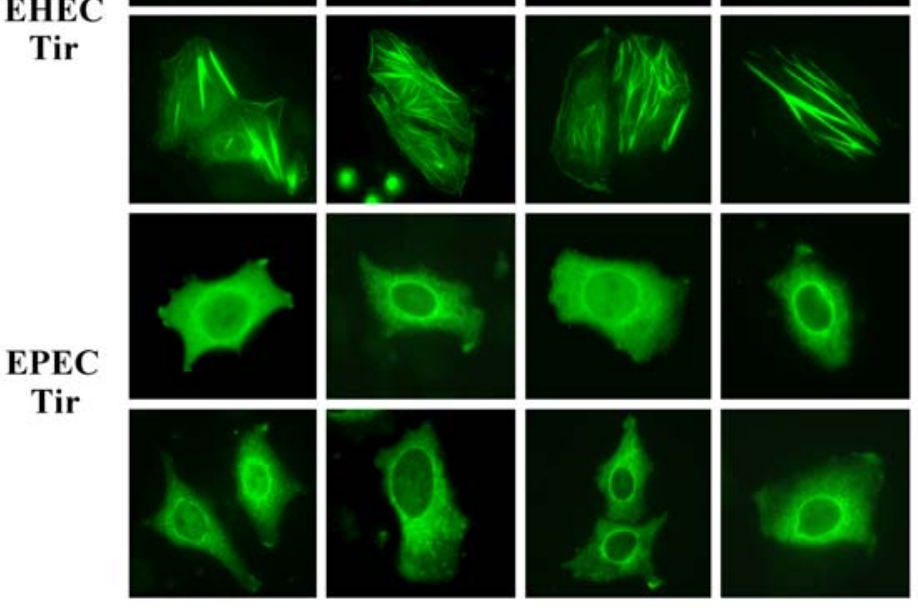

(b)
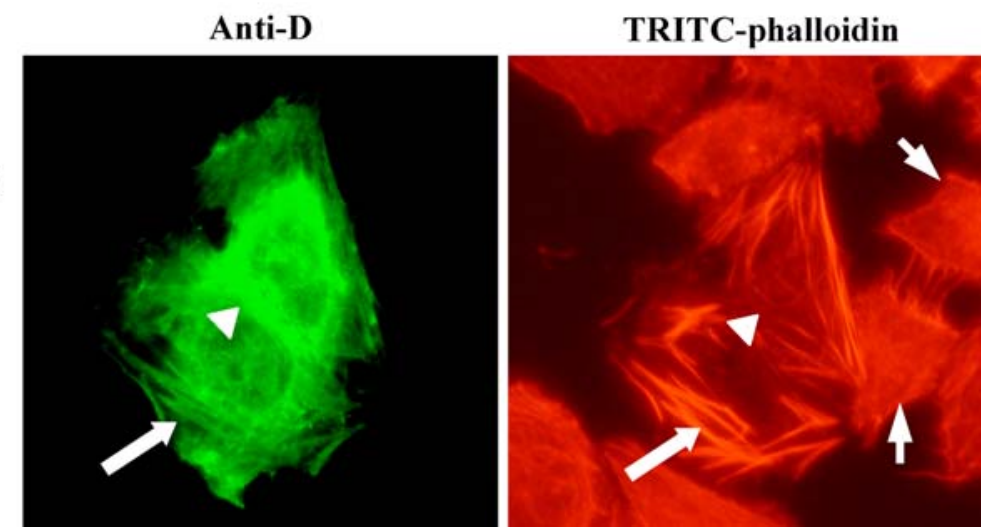

Figure 2. Localization of Tir expressed in HeLa cells by immunofluorescence staining. After transfection for $48 \mathrm{~h}$, cells on cover slips were processed for immunostaining. (a) Cells were fixed with methanol/acetone before reacting with antibodies. The primary antibodies used were mouse MAb to the tag on Tir, which was in turn followed by FITC-conjugated goat anti-mouse IgG. The D epitope tagged at the N-terminus of Tir was detected by HP6A1 whereas the C-terminal M epitope was stained with SC1D7. Images were taken at $100 \times$ magnification. (b) Comparison of fibrous structures within the cells doubly stained for EHEC Tir and actin. Staining EHEC Tir was done as in (a) using anti-D MAb whereas actin was stained with TRITC-labeled phalloidin. Long arrow indicates the superimposable fibrous structures, arrowhead labels those that are non-superimposable whereas short arrows mark representative cells without expressing EHEC Tir. Images were taken at $400 \times$ magnification.

transfection were prepared in an appropriate buffer and clarified first by centrifugation at $13,000 \mathrm{~g}$. Then, the clarified supernatants were gently shaken at $4^{\circ} \mathrm{C}$ for $14 \mathrm{~h}$. Intriguingly, it was observed that the cell lysate derived from the expression of EHEC Tir had more insoluble material than its counterparts derived from EPEC Tir or from a vector control. After a brief centrifugation and rinsing with the same buffer, proteins in the pellets were analyzed with SDSPAGE followed by Coomassie blue dye staining. Figure $3 \mathrm{a}$ shows that before the incubation all samples contained comparable amounts of proteins (lanes 1-3). However, after the incubation, the pellet originated from EHEC Tir-expressing cells apparently contained more proteins than the other two samples (compare lane 4 with lanes 5 and 6). In particular, a $43-\mathrm{kDa}$ protein and a few 
proteins with sizes smaller than $18 \mathrm{kDa}$ were enriched.

The 43-kDa protein seen in Figure 3a (lane 4) has a size that coincides with that of actin, which is known to polymerize in vitro when conditions are appropriate. It was also observed above that the F-actin structures stained were thicker in the EHEC Tir-expressing cells than in those without Tir expression (see Figure 2b). Therefore, the 43-kDa extra protein found could possibly be the actin molecule. To investigate this possibility, the insoluble pellets were thoroughly washed. This procedure was facilitated by adding to the cell lysates anti-M MAb that was pre-bound to protein A-conjugated agarose during the 14-h incubation. After incubation, the insoluble pellets along with the protein A-agarose/anti-M/Tir complexes were washed thoroughly with the incubation buffer. The proteins in the pellets were then dissolved in the SDS sample buffer and analyzed for the presence of the Tir molecules by Western blotting using anti-D MAb (Figure 3b, left panel). As expected, Tir molecules of both EHEC and EPEC origins were detected. The presence of the 43-kDa protein, which is smaller than the heavy chain of immunoglobin, could be seen in the sample prepared from the EHEC Tir-expressing cells, but not in that derived from the EPEC counterpart (Figure 3b, right panel). This result was consistent with that observed in Figure 3a. To deduce the identity of this $43-\mathrm{kDa}$ protein, the band in the gel was cut out and subjected to an in-gel trypsin digestion. The eluted peptides were analyzed by ESI-Q-TOF mass spectrometry. Figure $3 \mathrm{c}$ shows the results of the peptides deduced. A high degree of the peptide coverage resulted in the conclusion that the 43$\mathrm{kDa}$ protein was actin.

\section{Non-phosphorylated Tir from both EHEC and EPEC}

To characterize the molecular properties of Tir synthesized intracellularly further, a comparison was made between molecules generated by infection and transfection (Figure 4). Tir from cells infected with EHEC was detected as three different sizes by anti-EHEC-Tir (lane 1). After treatment with alkaline phosphatase (AP), the upper two bands disappeared, and only the lowest band remained detected (lane 2 ), a result suggesting that the former includes phosphorylated products (a)

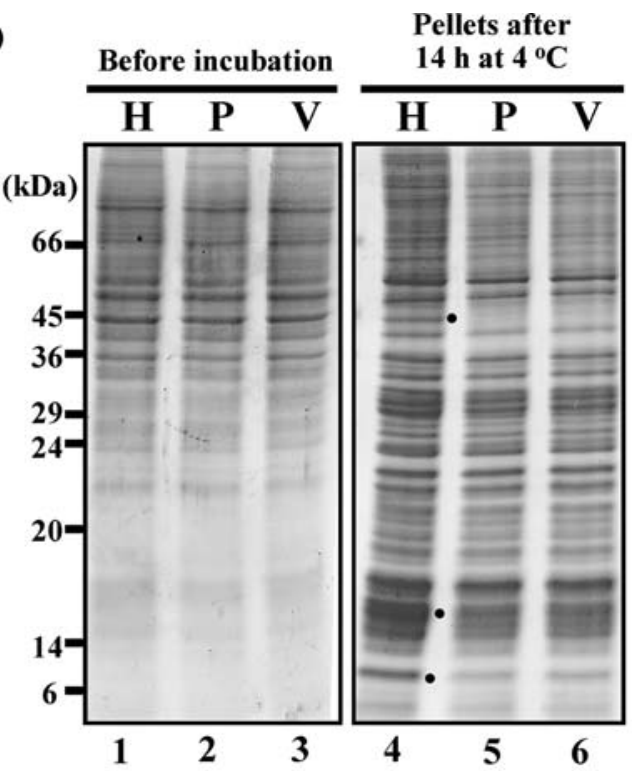

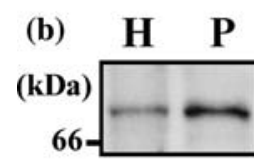

Anti-D

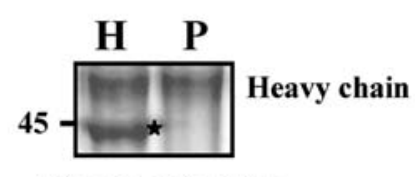

Coomassie blue (c) MDDDIAALVV DNGSGMCKAG FAGDDAPRAV FPSIVGRPRH QGVMVGMGQK DSYVGDEAQS KRGILTLKYP IEHGIVTNWD DMEKIWHHTF YNELRVAPEE HPVLLTEAPL NPKANREKMT QIMFETFNTP AMYVAIQAVL SLYASGRTTG IVMDSGDGVT HTVPIYEGYA LPHAILRLDL AGRDLTDYLM KILTERGYSF TTTAEREIVR DIKEKLCYVA LDFEQEMATA ASSSSLEKSY ELPDGQVITI GNERFRCPEA LFQPSFLGME SCGIHETTFN SIMKCDVDIR KDLYANTVLS GGTTMYPGIA DRMQKEITAL APSTMKIKII APPERKYSVW IGGSILASLS TFQQMWISKQ EYDESGPSIV HRKCF*

Figure 3. Molecular aggregation promoted by EHEC Tir. Cell lysates were centrifuged at $13,000 \mathrm{~g}$ for $10 \mathrm{~min}$. The supernatants were further incubated at $4^{\circ} \mathrm{C}$ for $14 \mathrm{~h}$. Insoluble structures formed during the incubation period were pelleted and analyzed. Transfected plasmids were: $\mathrm{H}, \mathrm{pC} / \mathrm{d}-\mathrm{Tir}-\mathrm{m}-\mathrm{H}$; $\mathrm{P}, \mathrm{pC} / \mathrm{d}$-Tir-m-P; V, control vector pCMV-DD6. (a) Proteins separated on SDS-PAGE and stained with Coomassie blue. Dots label protein bands stained to obviously different intensities among samples. (b) The cell lysates were incubated with anti-D/protein A Sepharose at $4^{\circ} \mathrm{C}$ for $14 \mathrm{~h}$ and then spun as in (a). Pellets were thoroughly washed with the same buffer at $4^{\circ} \mathrm{C}$ and dissolved in SDS sample buffer. Proteins were analyzed for Tir by Western blotting using anti-D MAb or directly stained on polyacrylamide gel with Coomassie blue. Band asterisked was eluted and subjected to ESI-Q-TOF mass spectrometry analysis. (c) Peptides (hatched) identified by ESI-Q-TOF are found within the known actin sequence. 


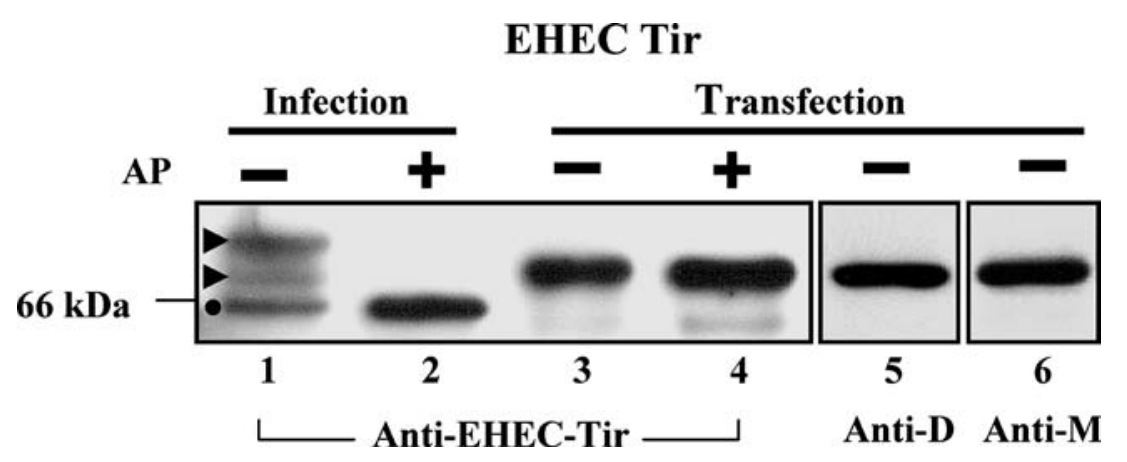

Figure 4. Comparison of phosphorylation between bacterial translocated Tir and transfection-expressed Tir. HeLa cells were infected with bacteria for $8 \mathrm{~h}$, and cells were directly lysed in the SDS sample buffer. Transfection was carried out with pC/d-Tir-m$\mathrm{H}$, and the cells were further cultivated for $48 \mathrm{~h}$. Cells were washed twice with PBS and lysed by freeze and thaw. Cell lysates were directly mixed with SDS sample buffer or treated with calf intestine phosphatase (AP) first for 30 min and then mixed with SDS sample buffer. Samples were then analyzed by Western blotting using different antibodies. Note: Tir expressed from transfection was tagged on both ends with the D- and M-epitopes. As a result, its mobility on SDS-PAGE was slower than the unmodified Tir from EHEC. Dot labels the unmodified Tir whereas arrowheads mark those phosphorylated to different degrees.

whereas the latter must be non-phosphorylated Tir [13]. In contrast, d-Tir-m-H generated from transfection was seen as a single product (lane 3) and remained at the same gel mobility after AP digestion (lane 4). This phenomenon was seen consistently irrespective of which antibodies used (compare lanes 4-6). When the products of Tir from infection with EPEC were examined, phosphorylation at $\mathrm{Tyr}$ as reported previously [13] was consistently observed because of a positive reactivity of Tir with anti-PY monoclonal antibody (data not shown). However, no phosphorylation was observed with d-Tir-m-P when expressed by transfection. Therefore, Tir molecules from both EHEC and EPEC resulting from transfection do not serve as a substrate for intracellular phosphorylation, and in this regard, these two Tir molecules behaved similarly.

\section{Mapping the region of EHEC Tir critical for fibrous structure formation in cells}

The data in Figure $2 b$ indicated that d-Tir-m of EHEC has the ability to form fibrous structures, and apparently neither phosphorylation nor additional bacterial effectors are required for this cellular event. The counterpart from EPEC was not phosphorylated either, but it has no such a capacity. To deduce which structural characteristic causes this unique property of EHEC Tir, deletion was first used (Figure 5) to map the critical region of the molecule. Before mapping, the effect of the tags on the presentation of Tir must be clarified.
Constructs d-Tir-H and Tir-m-H remove the $\mathrm{M}$ tag from the $\mathrm{C}$-terminus and the $\mathrm{D}$ tag from the $\mathrm{N}$ terminus of d-Tir-m-H, respectively. Both products, when immunofluorescence was used to stain, gave patterns in cells similar to that observed for d-Tir$\mathrm{m}-\mathrm{H}$ (Figure 5). Therefore, the tags did not affect the molecular presentation of EHEC Tir in the cells. Additional deletion mutants were then created, and their products in cells were similarly examined. Constructs preserving the $\mathrm{N}$-terminal two thirds (in d-Tir ${ }_{\mathrm{H} 1-390}$ ) or the middle one third (in d-Tir ${ }_{\mathrm{H} 228-390}$ ) were observed diffusely distributed in the cytosol, and no fibrous immunofluorescence pattern was observed. However, in the construct $\mathrm{d}-\mathrm{Tir}_{\mathrm{H} 228-558}-\mathrm{m}$, the stained fibrous pattern of the product was indistinguishable from that of d-Tir-m-H. Thus, the N-terminal first 227 amino acids were not critical for this fibrous structure formation, and the motif conferring the fibrous distribution ought to be in the C-terminal region of EHEC Tir, likely after residue 390.

Since deletion may result in a drastic structural change and since two homologous but distinct molecules in EHEC and EPEC are available, a fragment swapping experiment was performed. First, the N-terminal region of EPEC Tir was fused to the C-terminal fragment of EHEC Tir to produce a series of chimera with the same length as the authentic EPEC Tir, i.e. a total of 550 amino acid residues (Figure 6a). In $\mathrm{d}-\mathrm{Tir}_{\mathrm{P} 528 \mathrm{H}^{-}} \mathrm{m}$, the last 22 residues of EPEC Tir were replaced by counterparts from the EHEC Tir. The chimeric Tir was observed mostly with the diffusive pattern in the 


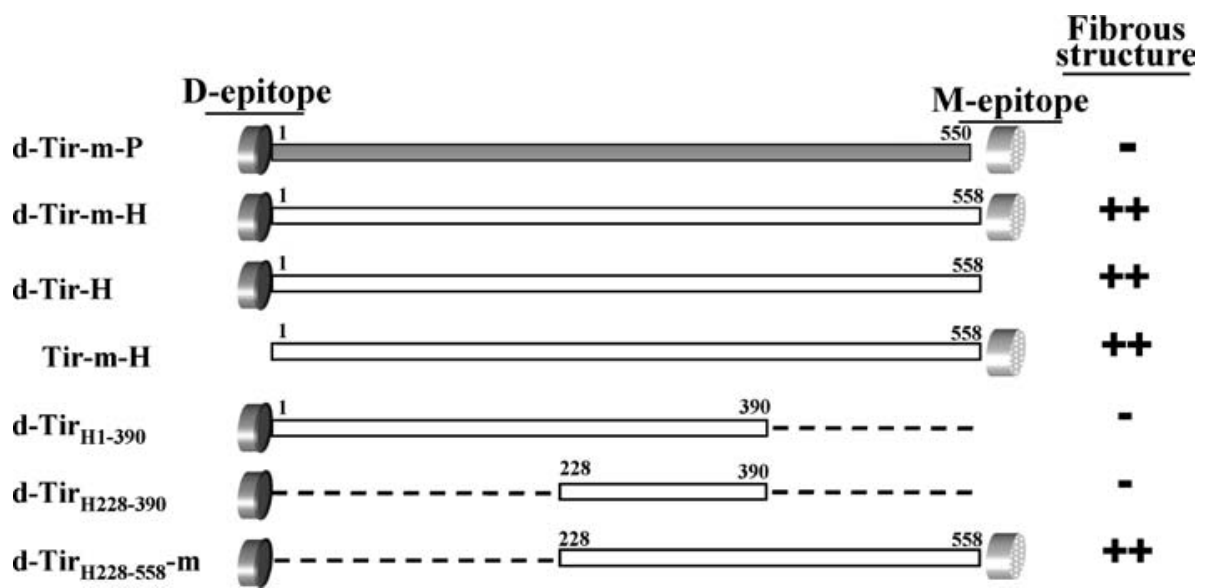

Figure 5. Mapping the region of EHEC Tir responsible for the formation of fibrous structure. Cells were transfected with various constructs of Tir and stained by anti-D (or anti-M) followed by FITC-goat-anti-mouse IgG. The staining patterns of transfected cells were observed under immunofluorescence microscope and scored for cells with the filamentous pattern. As a control, d-Tir-m$\mathrm{P}$ gave no cell showing fibrous structure among 217 Tir-expressing cells that were counted $(0 / 217)$ whereas d-Tir-m-H gave a frequency of $41 \%$ among the cells counted (86/208). Deleting either D- or M-epitope from the parental constructs gave results similar to d-Tir-m-H. Effects of other deletions on the fibrous formation are summarized as follows: ++ , more than $20 \%$ of the Tirexpressing cells stained in a filamentous pattern; -, no fibrous structure observed with at least 200 Tir-expressing cells. Dashed line within a construct represents where a region is deleted; numbers labeled above the bar are amino acid residues in EHEC Tir.

cytoplasm, but occasionally $(3.6 \%)$ as the fibrous structures. By fusing the last 38 residues of EHEC Tir with the N-terminal 512 residues of EPEC Tir in $\mathrm{d}-\operatorname{Tir}_{\mathrm{P} 512 \mathrm{H}^{-}} \mathrm{m}$, the chimeric molecule was seen to have a fibrous structure pattern at a frequency of about $10 \%$. Apparently, the positive rate with d-Tir ${ }_{\mathrm{P} 512 \mathrm{H}^{-} \mathrm{m}}$ was higher than with d-Tir ${ }_{\mathrm{P} 528 \mathrm{H}^{-}}-\mathrm{m}$. Including more residues from the $\mathrm{C}$-terminal portion of EHEC Tir and decreasing more residues from the $\mathrm{N}$-terminal portion of EPEC Tir in the chimera, i.e. d- $\operatorname{Tir}_{\mathrm{P}_{3} 75 \mathrm{H}^{-} \mathrm{m}}$, further increased the frequencies of observed Tir with the fibrous pattern (Figure 6a). Further incorporation of more C-terminal residues from EHEC Tir to the chimera (in $\mathrm{d}-\operatorname{Tir}_{\mathrm{P} 173 \mathrm{H}^{-}}-\mathrm{m}$ ), however, did not increase the frequency of observed fibrous structure. Therefore, to effectively present fibrous structures by chimeric Tir, the C-terminal region after residue 374 of EHEC appeared to be sufficient, a conclusion similar to that derived from fragment deletions in Figure 5, in which the importance of the region after residue 390 is suggested.

By doing reciprocal experiments that fused the N-terminal fragment of EHEC Tir with the C-terminal fragment of EPEC, the replaceable region of EHEC Tir could be defined (Figure 6a). In the constructs $\mathrm{d}-\mathrm{Tir}_{\mathrm{H} 171 \mathrm{P}}-\mathrm{m}$ and $\mathrm{d}-\mathrm{Tir}_{\mathrm{H} 376 \mathrm{P}}$, the C-terminal critical region of EHEC Tir (after residue 390) as deduced above in Figure 6a was replaced by the swapping. Therefore, they were expected to give a diffusive distribution pattern, and, indeed, the results were as expected

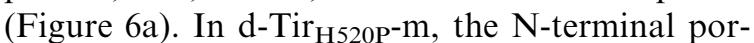
tion up to residue 520 of the chimera was derived from EHEC Tir, and only the last 38 residues were replaced with their counterparts from EPEC. Strikingly, the immunofluorescence-stained Tir was found diffusely in the cytoplasm, and thus the last 38 residues of EHEC Tir are important. Therefore, swapping was further narrowed down to the last five residues of Tir, in which only SerHis at residues 554 and 555 are nonconservatively changed to Pro-Val (Figure 6c), and the resulting $\mathrm{d}-\mathrm{Tir}_{\mathrm{H} 553 \mathrm{P}} \mathrm{-m}$ was stained fibrously with a frequency similar to that of the parental d-Tir-m-H (Figure 6a). Therefore, variations within the last five C-terminal residues of EHEC Tir are not critical to the fibrous pattern presentation.

To determine whether the last 38 residues of EHEC Tir could be divided into small fragments and swapped with those from EPEC, two additional constructs were created (Figure 6a).

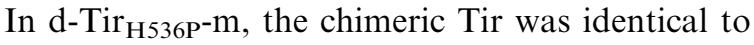
$\mathrm{d}-\mathrm{Tir}-\mathrm{m}-\mathrm{H}$ except that the residues after position 537 were replaced with residues $529-550$ of the EPEC Tir; as a result, residues at 11 positions differ from those of EHEC Tir (Figure 6c). In the 
(a)

$\mid \begin{aligned} & \text { d-Tir }{ }_{\mathrm{P} 528 \mathrm{H}^{-m}} \\ & \text { d-Tir } \\ & \text { d-Tir } \operatorname{Tir}_{\mathrm{P} 375 \mathrm{H}^{-m}}-\mathrm{m} \\ & \text { d-Tir } \\ & {\mathrm{P} 173 \mathrm{H}^{-m}}^{-m}\end{aligned}$

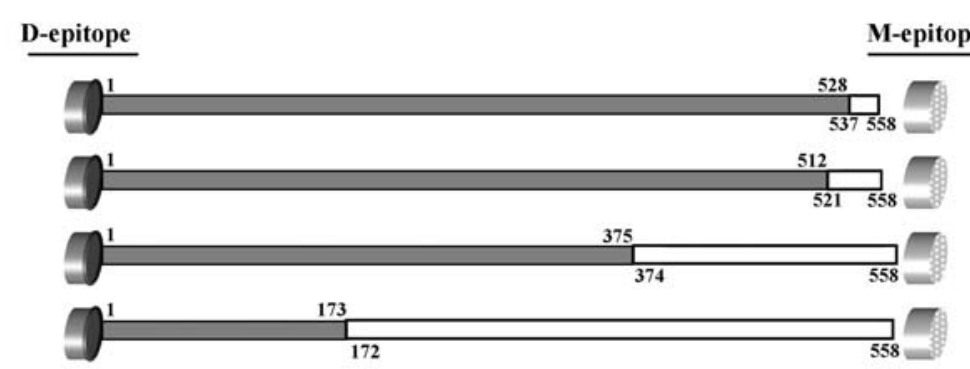

Fibrous

$\mid \begin{aligned} & \text { d-Tir }{ }_{\mathrm{H} 171 \mathrm{P}}-\mathrm{m} \\ & \text { d-Tir }{ }_{\mathrm{H} 376 \mathrm{P}} \\ & \text { d-Tir }{ }_{\mathrm{H} 520 \mathrm{P}}-\mathrm{m} \\ & \text { d-Tir }{ }_{\mathrm{H} 553 \mathrm{P}}-\mathrm{m} \\ & \text { d-Tir }{ }_{\mathrm{H} 536 \mathrm{P}^{-m}} \\ & \text { d-Tir }{ }_{\mathrm{H} 520 \mathrm{P532H}}-\mathrm{m}\end{aligned}$

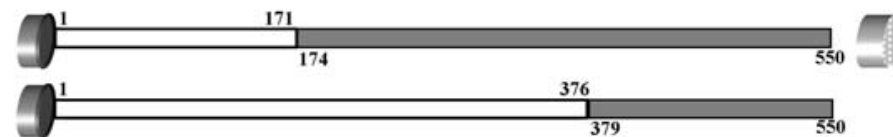

- NC

- NC

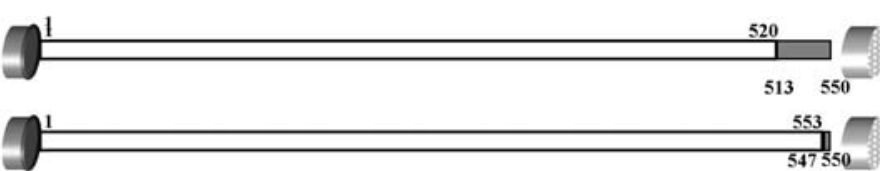

- $\quad 0 / 224(0 \%)$

$++\mathrm{NC}$

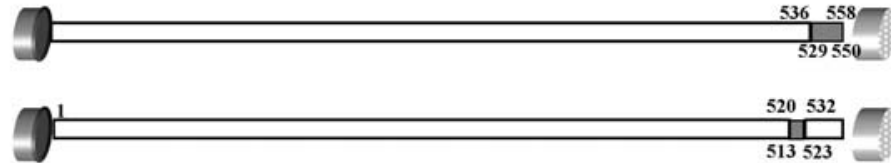

$+72 / 213(34 \%)$

$+54 / 228(24 \%)$

(b)

d-Tir ${ }_{\mathrm{H} 497-558}-\mathrm{m}$

(c)

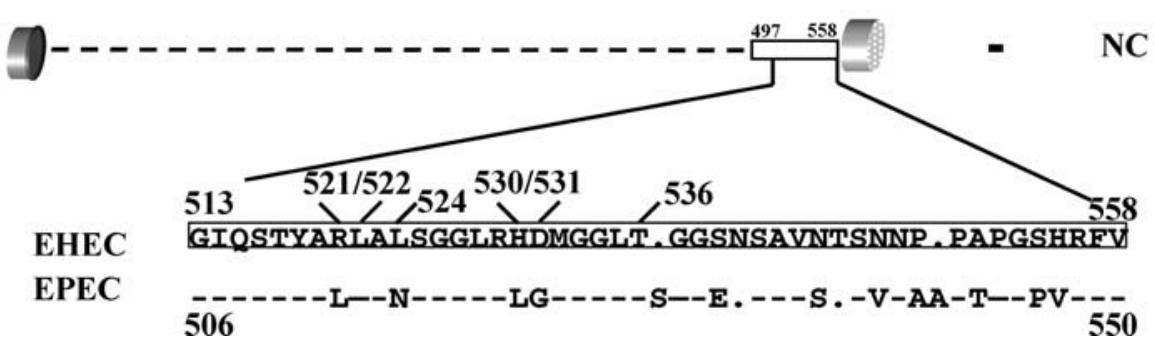

Figure 6. Confirming the C-terminal region of EHEC Tir critical for the fibrous structure formation in cells. HeLa cells were transfected with different constructs, and the expressed Tir was immunostained with anti-D as in Figure 5. Patterns of Tir stained were observed under a fluorescence microscope, and cells presenting filamentous structure were enumerated among the Tir-expressing cells. (a) Constructs with fragment swapped between the Tir molecules of EPEC and EHEC. Hatched bar represents the fragment derived from EPEC Tir whereas open bar indicates the fragment swapped from EHEC Tir; numbers labeled above the bar are residues in EHEC Tir whereas those in EPEC Tir are placed underneath. (b) Construct comprising the last 68 C-terminal residues of EHEC Tir. See legend to Figure 5 for the explanations of ++ and - for those samples not precisely counted (NC). (c) A blownup C-terminal region of EHEC Tir. Residues different from EPEC Tir are labeled; those identical are represented by dashes; gaps were introduced to maximize the alignment and are denoted by dots.

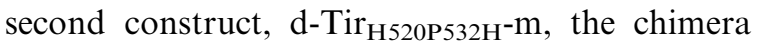
was identical to d-Tir-m-H except that residues 521, 524, 530 and 531 are those from the EPEC Tir. Results from immunofluorescence staining indicated that these chimeric Tir molecules gave both patterns of diffusive distribution and fibrous structures in cytoplasm. The frequencies of fibrous structure-forming cells were scored at $24 \%$ and $34 \%$ for $\mathrm{d}-\mathrm{Tir}_{\mathrm{H} 520 \mathrm{P} 532 \mathrm{H}}-\mathrm{m}$ and $\mathrm{d}-\mathrm{Tir}_{\mathrm{H} 536 \mathrm{P}} \mathrm{m}$, respectively, and both values were slightly lower than the rate $(41 \%)$ observed with the wild type molecule. Thus, these limited changes did not abolish the fibrous structure forming activity of EHEC Tir but did slightly decrease the efficiency. Therefore, swapping a small portion at the C-terminal extreme region may be tolerable while an extensive replacement definitely abolishes the activity of EHEC Tir to form the fibrous structures.

The above observations with $\mathrm{d}-\mathrm{Tir}_{\mathrm{H} 520 \mathrm{P}}-\mathrm{m}$ indicated the importance of the $\mathrm{C}$-terminal last 
38 residues of the EHEC Tir in forming the fibrous structure. On the other hand, the result with d$\operatorname{Tir}_{\mathrm{P}_{512 \mathrm{H}}}-\mathrm{m}$ suggested that the 38 -residue C-terminal fragment of EHEC Tir in the context of the EPEC sequence might change the properties of EPEC Tir but only to one fourth of the frequency seen with an authentic EHEC Tir (i.e. 10\% vs. $41 \%$ ). Therefore, the contribution of the sequence upstream to the last 38-residue region is speculative. The protein d-Tir ${ }_{\mathrm{H} 497-558}-\mathrm{m}$ (Figure 6b) was constructed to extend the coverage and contains most of the C-terminal conservative region (Figure 1). However, this product was still detected exclusively in a diffusive pattern in the cytoplasm (Figure 6b). Therefore, the C-terminal region of EHEC Tir, up to 62 residues in length, is not sufficient to confer the property of displaying fibrous structures. Apparently, diverse residues Nterminally proximal to this C-terminal conserved region, i.e. residues 390-496 of EHEC Tir, may also play an auxiliary role.

The importance of the Tir C-terminal 38residue region on the EHEC pedestal formation (Figure 7a) was then substantiated by the bacterial infection assay. To do this, an EHEC tir-deleted mutant $(\Delta$ Tir) was generated. This mutant did not trigger pedestal formation when it encountered cells (Figure 7b). After complementation with pQE60-Tir-H, the bacteria restored the phenotype of pedestal formation seen with the wild type strain (compare Figure 7a and c). However, when the same bacteria were complemented with pQE60-Tir ${ }_{\mathrm{H} 1-521}$, no pedestal formation was seen (Figure 7d), a fact supporting the importance of the last 38 residues of EHEC Tir in forming pedestal.

\section{Discussion}

The homologous Tir molecules from EHEC and EPEC vary in their mechanisms during pedestal formation [19]. In order to understand their molecular differences, we used transfection experiments expressing these homologues in HeLa cells and obtained several findings. First, irrespective of the origin, these two Tir molecules readily share a common property: EHEC Tir is not phosphorylated when expressed by transfection, and neither is EPEC Tir (see above; [24]). Second, EHEC Tir differs from EPEC Tir in its ability to stimulate the formation of precipitants containing actin in vitro. Third, the actin filaments stained within the cells expressed EHEC Tir are thicker than those in cells without transfection (see Figure 2b) or expressing EPEC Tir (not shown). Finally and most strikingly, a fibrous pattern is seen with EHEC Tir, but not with that of EPEC, and this property has been experimentally demonstrated to associate with the unique C-terminal sequence of EHEC Tir.

In EPEC, the tyrosine-phosphorylated Tir binds to the host cell adaptor protein Nck [31], which in turn results in the recruitment of N-WASP and the Arp2/3 actin-nucleating machinery required for the pedestal formation [20]. Accordingly, recruitment of actin, VASP, and N-WASP is abolished in the absence of this tyrosine phosphorylation [31]. Since our Tir molecule resulted from transfection is not phosphorylated, it is then not surprising to see our precipitant from EPEC Tir does not contain actin molecules. Conversely, actin was detected in the pellet that contained the non-phosphorylated EHEC Tir. This result is consistent with the concept that EHEC's reorganization of the cytoskeleton is in a tyrosine phosphorylation independent manner [32]. Since the pedestal formation is mediated through multiple steps of protein recruitment and activation [20,33] that lead to a final filament condensation, presence of actin and Tir in the same complexes may not necessarily represent a direct protein-protein interaction. Instead, the EHEC Tir may facilitate actin assembly indirectly [19], and this capacity may not solely depend on $\operatorname{EspF}_{\mathrm{U}}$, a product encoded within the EHEC prophage-U that has been recently found to bind directly to N-WASP [21]. $\mathrm{EspF}_{\mathrm{U}}$ has also been named TccP, and no binding of TccP with EHEC Tir has been detected [34].

Moving towards the elucidation of why EHEC Tir has no Tyr 474 but eventually induces similar pedestal formation like EPEC Tir, we have found that EHEC Tir, but not that of EPEC, has the unique capability of forming fibrous structure in cells. This property has been observed with a minimal fragment of the C-terminal region containing residues 228-558 of EHEC Tir (see Figure 5). Since residues 228-390 are highly conserved between EHEC Tir and EPEC Tir and since this fragment was experimentally shown without this property (see Figure 5), it strongly suggested that EHEC Tir after residue 390 might comprise 


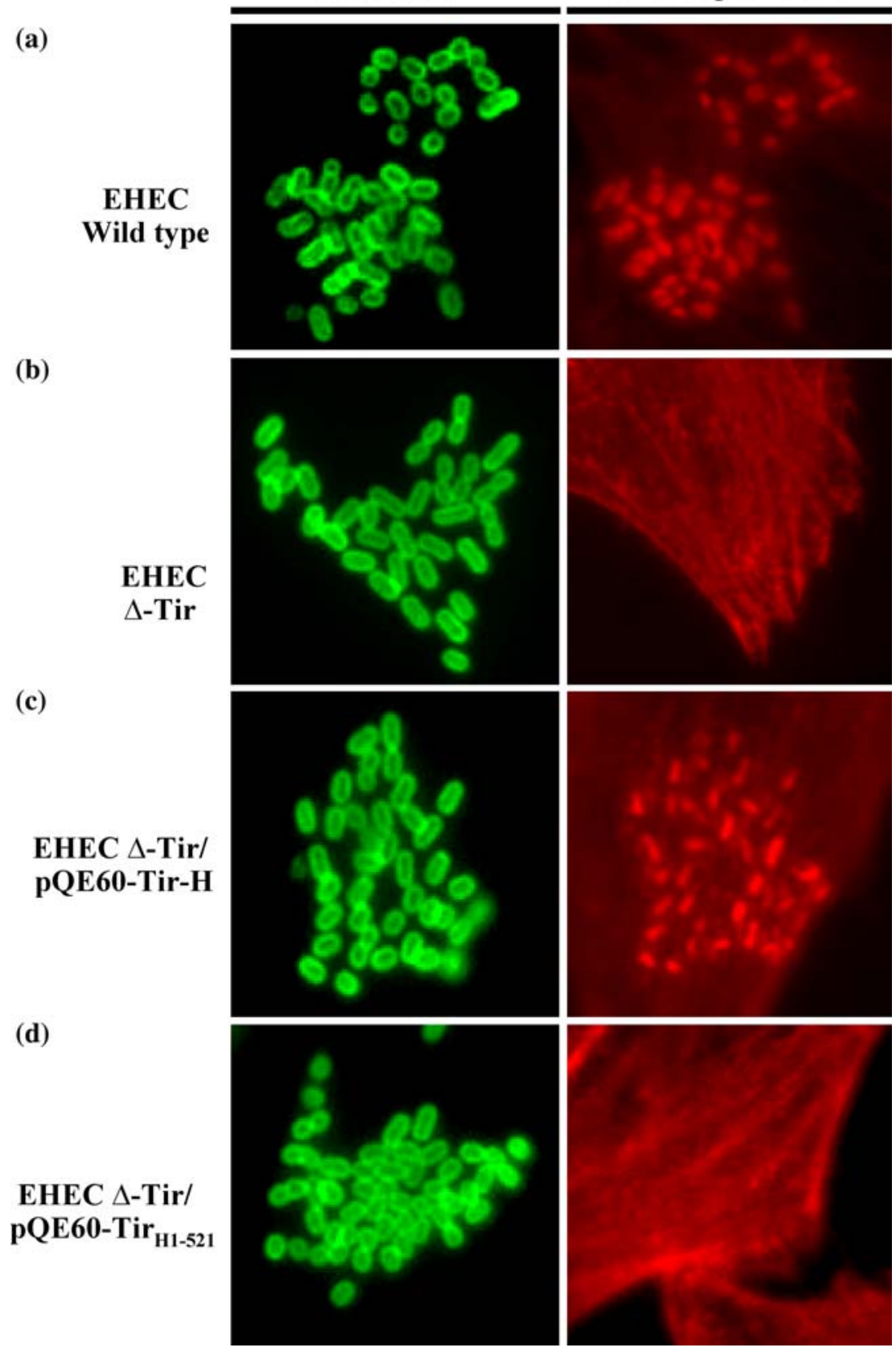

Figure 7. EHEC pedestal formation abolished by deleting the C-terminal 38 residues of Tir. HeLa cells were infected with bacteria and then washed with PBS. After washings, cultured cells were fixed with methanol/acetone and simultaneously reacted with TRITC-phalloidin and rabbit anti-O157. The bacteria-bound anti-O157 was further detected by FITC-conjugated goat anti-rabbit IgG. (a) The parental strain of EHEC; (b) $\Delta$ Tir, a tir-deleted EHEC mutant; (c) $\Delta$ Tir transformed with pQE60-Tir-H; (d) $\Delta$ Tir transformed with $\mathrm{pQE60-Tir} \mathrm{H}_{1-521}$

the major determinant. Data from the fragment swapping in Figure 6 further supports this notion. Taking together, we conclude that this unique property of EHEC Tir is inherited in residues 390-553. This C-terminal region of Tir contains the most divergent sequence and a downstream semi-conservative stretch between the two Tir molecules. To perform the unique 
function of EHEC Tir, the semi-conservative residues is speculated to provide a basic framework while the varied residues may assist the formation of the distinctive structure.

In a live imaging of the pedestal formation when EHEC encounters a host cell, our preliminary results showed that a total of about $6 \mathrm{~h}$ was required. During this period, many interactions among the bacterial and host cellular molecules must occur. Although our transfection system oversimplifies the events during the bacteria-cell contact, it may offer a unique opportunity to observe the intrinsic properties associated with Tir that is not otherwise easily revealed by the infection system. For instance, Tir from an EPEC-infected cell could be detected in the cytoplasm as well as on the membrane where Tir possesses a particular topological structure and is phosphorylated (de Grado et al. 1999). On the other hand, EPEC Tir produced from our transfection did not retain all these properties. Campellone et al. [21] has estimated that approximately one fifth of a similarly produced EPEC Tir reaches the membrane as compared to an ectopically engineered construct of Tir. EHEC Tir produced by transfection did behave similarly as seen above. Some of the molecules reached the membrane but most of them remained in the cytoplasm. Thus, a high concentration of EHEC Tir in the cytoplasm may account for the formation of the fibrous structure that could not be seen during the infection.

Toward condensation of the actin filaments and formation of EHEC pedestal structure, TccP present only in EHEC must be translocated into the hose cell. Moreover, a putative factor of a cellular origin must also be recruited [34] to link between Tir and TccP. It is then speculated that this indirect linking of EHEC Tir to TccP may not be as efficient as the route used by EPEC $\operatorname{Tir}\left(\mathrm{Tyr}_{474}\right)$-Nck. If so, the fiber formation of EHEC Tir could be physiologically relevant since this clustering effect may facilitate the recruitment of signal transduction factors [24]. A possibility of the fiber formation due to an over-expression of Tir is less likely since EPEC Tir similarly expressed did not yield the same result.

Nevertheless, the importance of the last 38 residues of EHEC Tir observed in the fibrous structure (see Figure 6a) was also seen with the pedestal formation in the bacterial infection (see Figure 7). The distinct $\mathrm{C}$-terminal region with less than one third of the Tir molecule therefore must bear rich information crucial for signaling. What the molecule could be to bridge between EHEC Tir and TccP and whether it interacts with EHEC Tir in the identified C-terminal region are warranted for further exploration.

\section{Acknowledgements}

This research was supported in parts by Grant 89-B-FA22-2-4 (Program for Promoting Academic Excellence of Universities) from the Department of Education and NSC 92-2320B-010-048 from the National Science Council, Taiwan, R.O.C.

\section{References}

1. Nataro J.P. and Kaper J.B., Diarrheagenic Escherichia coli. Clin. Microbiol. Rev. 11: 142-201, 1998.

2. Frankel G., Phillips A.D., Rosenshine I., Dougan G., Kaper J.B. and Knutton S., Enteropathogenic and enterohaemorrhagic Escherichia coli: more subversive elements. Mol. Microbiol. 30: 911-921, 1998.

3. McDaniel T.K. and Kaper J.B., A cloned pathogenicity island from enteropathogenic Escherichia coli confers the attaching and effacing phenotype on E. coli K-12. Mol. Microbiol. 23: 399-407, 1997.

4. Perna N.T., Mayhew G.F., Posfai G., Elliott S., Donnenberg M.S., Kaper J.B. and Blattner F.R., Molecular evolution of a pathogenicity island from enterohemorrhagic Escherichia coli O157:H7. Infect. Immun. 66: 3810-3817, 1998.

5. Elliott S.J., Wainwright L.A., McDaniel T.K., Jarvis K.G., Deng Y.K., Lai L.C., McNamara B.P., Donnenberg M.S. and Kaper J.B., The complete sequence of the locus of enterocyte effacement (LEE) from enteropathogenic Escherichia coli E2348/69. Mol. Microbiol. 28: 1-4, 1998.

6. Kenny B., DeVinney R., Stein M., Reinscheid D.J., Frey E.A. and Finlay B.B., Enteropathogenic E. coli (EPEC) transfers its receptor for intimate adherence into mammalian cells. Cell 91: 511-520, 1997.

7. Hartland E.L., Batchelor M., Delahay R.M., Hale C., Matthews S., Dougan G., Knutton S., Connerton I. and Frankel G., Binding of intimin from enteropathogenic Escherichia coli to Tir and to host cells. Mol. Microbiol. 32: 151-158, 1999.

8. Kenny B., Lai L.C., Finlay B.B. and Donnenberg M.S., EspA, a protein secreted by enteropathogenic Escherichia coli, is required to induce signals in epithelial cells. Mol. Microbiol. 20: 313-323, 1996.

9. Donnenberg M.S., Yu J. and Kaper J.B., A second chromosomal gene necessary for intimate attachment of enteropathogenic Escherichia coli to epithelial cells. J. Bacteriol. 175: 4670-4680, 1993. 
10. Lai L.C., Wainwright L.A., Stone K.D. and Donnenberg M.S., A third secreted protein that is encoded by the enteropathogenic Escherichia coli pathogenicity island is required for transduction of signals and for attaching and effacing activities in host cells. Infect. Immun. 65: 22112217, 1997.

11. Crane J.K., McNamara B.P. and Donnenberg M.S., Role of EspF in host cell death induced by enteropathogenic Escherichia coli. Cell Microbiol. 3: 197-211, 2001.

12. Kenny B. and Jepson M., Targeting of an enteropathogenic Escherichia coli (EPEC) effector protein to host mitochondria. Cell Microbiol. 2: 579-590, 2000.

13. Kenny B., Phosphorylation of tyrosine 474 of the enteropathogenic Escherichia coli (EPEC) Tir receptor molecule is essential for actin nucleating activity and is preceded by additional host modifications. Mol. Microbiol. 31: 12291241, 1999.

14. Batchelor M., Guignot J., Patel A., Cummings N., Cleary J., Knutton S., Holden D.W., Connerton I. and Frankel G., Involvement of the intermediate filament protein cytokeratin-18 in actin pedestal formation during EPEC infection. EMBO Rep. 5: 104-110, 2004.

15. Goosney D.L., DeVinney R. and Finlay B.B., Recruitment of cytoskeletal and signaling proteins to enteropathogenic and enterohemorrhagic Escherichia coli pedestals. Infect. Immun. 69: 3315-3322, 2001.

16. Rohatgi R., Nollau P., Ho H.Y., Kirschner M.W. and Mayer B.J., Nck and phosphatidylinositol 4,5-bisphosphate synergistically activate actin polymerization through the $\mathrm{N}$ WASP-Arp2/3 pathway. J. Biol. Chem. 276: 26448-26452, 2001.

17. Campellone K.G., Giese A., Tipper D.J. and Leong J.M., A tyrosine-phosphorylated 12-amino-acid sequence of enteropathogenic Escherichia coli Tir binds the host adaptor protein Nck and is required for Nck localization to actin pedestals. Mol. Microbiol. 43: 1227-1241, 2002.

18. Kalman D., Weiner O.D., Goosney D.L., Sedat J.W., Finlay B.B., Abo A. and Bishop J.M., Enteropathogenic $E$. coli acts through WASP and Arp2/3 complex to form actin pedestals. Nat. Cell Biol. 1: 389-391, 1999.

19. Campellone K.G. and Leong J.M., Tails of two Tirs: actin pedestal formation by enteropathogenic $E$. coli and enterohemorrhagic E. coli O157:H7. Curr. Opin. Microbiol. 6: $82-90,2003$

20. Lommel S., Benesch S., Rottner K., Franz T., Wehland J. and Kuhn R., Actin pedestal formation by enteropathogenic Escherichia coli and intracellular motility of Shigella flexneri are abolished in N-WASP-defective cells. EMBO Rep. 2: 850-857, 2001.

21. Campellone K.G., Robbins D. and Leong J.M., EspFU is a translocated EHEC effector that interacts with Tir and N-WASP and promotes Nck-independent actin assembly. Dev. Cell 7: 217-228, 2004
22. Sambrook J. and Russell D., Molecular Cloning: A Laboratory Manual, 3rd ed., Cold Spring Harbor Laboratory Press, Cold Spring Harbor, 2001.

23. Chuang C.H., Hsu S.C., Hsu C.L., Hsu T.C. and Syu W.J., Construction of a tagging system for subcellular localization of proteins encoded by open reading frames. J. Biomed. Sci. 8: 170-175, 2001.

24. Campellone K.G., Rankin S., Pawson T., Kirschner M.W., Tipper D.J. and Leong J.M., Clustering of Nck by a 12residue Tir phosphopeptide is sufficient to trigger localized actin assembly. J. Cell. Biol. 164: 407-416, 2004.

25. Lin H.P., Hsu S.C., Wu J.C., Sheen I.J., Yan B.S. and Syu W.J., Localization of isoprenylated antigen of hepatitis delta virus by anti-farnesyl antibodies. J. Gen. Virol. 80: 91-96, 1999.

26. Hsu S.C., Lin H.P., Wu J.C., Ko K.L., Sheen I.J., Yan B.S., Chou C.K. and Syu W.J., Characterization of a strain-specific monoclonal antibody to hepatitis delta virus antigen. J. Virol. Methods 87: 53-62, 2000.

27. Knutton S., McConnell M.M., Rowe B. and McNeish A.S., Adhesion and ultrastructural properties of human enterotoxigenic Escherichia coli producing colonization factor antigens III and IV. Infect. Immun. 57: 3364-3371, 1989.

28. Phan-Thanh L. and Mahouin F., A proteomic approach to study the acid response in Listeria monocytogenes. Electrophoresis 20: 2214-2224, 1999.

29. Yu S.L., Ko K.L., Chen C.S., Chang Y.C. and Syu W.J., Characterization of the distal tail fiber locus and determination of the receptor for phage AR1, which specifically infects Escherichia coli O157:H7. J. Bacteriol. 182: 59625968, 2000.

30. Chiu H.J., Lin W.S. and Syu W.J., Type III secretion of EspB in enterohemorrhagic Escherichia coli O157:H7. Arch. Microbiol. 180: 218-226, 2003.

31. Gruenheid S., DeVinney R., Bladt F., Goosney D., Gelkop S., Gish G.D., Pawson T. and Finlay B.B., Enteropathogenic E. coli Tir binds Nck to initiate actin pedestal formation in host cells. Nat. Cell Biol. 3: 856-859, 2001.

32. DeVinney R., Stein M., Reinscheid D., Abe A., Ruschkowski S. and Finlay B.B., Enterohemorrhagic Escherichia coli $\mathrm{O} 157: \mathrm{H} 7$ produces Tir, which is translocated to the host cell membrane but is not tyrosine phosphorylated. Infect. Immun. 67: 2389-2398, 1999.

33. Matsuzawa T., Kuwae A., Yoshida S., Sasakawa C. and Abe A., Enteropathogenic Escherichia coli activates the RhoA signaling pathway via the stimulation of GEF-H1. EMBO J. 23: 3570-3582, 2004.

34. Garmendia J., Phillips A.D., Carlier M.F., Chong Y., Schüller S., Marches O., Dahan S., Oswald E., Shaw R.K., Knutton S. and Frankel G., TccP is an enterohaemorrhagic Escherichia coli $\mathrm{O} 157: \mathrm{H} 7$ type III effector protein that couples Tir to the actin-cytoskeleton. Cell Microbiol. 6: $1167-1183,2004$ 